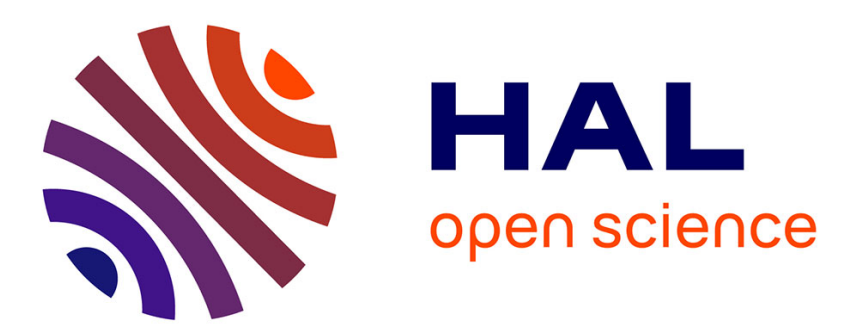

\title{
Calorimetric and thermodynamic study of glass-forming monohydroxy alcohols
}

\author{
Miguel Angel Ramos, Bisher Kabtoul, Merzak Hassaine
}

\section{To cite this version:}

Miguel Angel Ramos, Bisher Kabtoul, Merzak Hassaine. Calorimetric and thermodynamic study of glass-forming monohydroxy alcohols. Philosophical Magazine, 2010, pp.1. 10.1080/14786435.2010.526649 . hal-00641193

\section{HAL Id: hal-00641193 https://hal.science/hal-00641193}

Submitted on 15 Nov 2011

HAL is a multi-disciplinary open access archive for the deposit and dissemination of scientific research documents, whether they are published or not. The documents may come from teaching and research institutions in France or abroad, or from public or private research centers.
L'archive ouverte pluridisciplinaire HAL, est destinée au dépôt et à la diffusion de documents scientifiques de niveau recherche, publiés ou non, émanant des établissements d'enseignement et de recherche français ou étrangers, des laboratoires publics ou privés. 


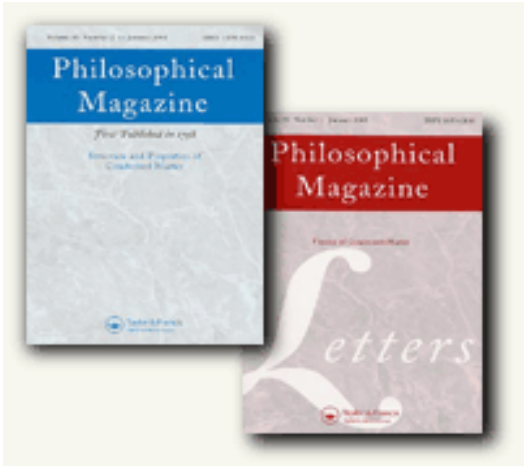

\section{Calorimetric and thermodynamic study of glass-forming monohydroxy alcohols}

\begin{tabular}{|r|l|}
\hline Journal: & Philosophical Magazine \& Philosophical Magazine Letters \\
\hline Manuscript ID: & TPHM-10-Jun-0283.R1 \\
\hline Journal Selection: & Philosophical Magazine \\
\hline Author: & 24-Aug-2010 \\
\hline & $\begin{array}{l}\text { Complete List of Authors: } \\
\text { Kabtoul, Bisher; Universidad Autonoma Madrid, Fisica de la Materia } \\
\text { Condensada } \\
\text { Hassaine, Merzak; Universidad Autonoma Madrid, Fisica de la } \\
\text { Materia Condensada }\end{array}$ \\
\hline Keywords: & $\begin{array}{l}\text { glass transition, specific heat, polymorphism, boson peak, } \\
\text { crystallization }\end{array}$ \\
\hline Keywords (user supplied): & monohydroxy alcohols, molecular glasses \\
\hline &
\end{tabular}

\section{SCHOLARONE ${ }^{\text {m }}$ \\ Manuscripts}




\begin{abstract}
In this work we review and concurrently discuss earlier and recent experiments performed on simple aliphatic glass-forming monoalcohols at low temperatures. By doing this, we find an interesting model system to explore different relevant issues concerning molecular glassforming liquids and even the glass transition phenomenon itself. They include the role played by the molecular aspect ratio in vitrification/crystallization kinetics, the reported appearance of particular cases of polymorphism and polyamorphism, and the influence of chemical isomerism and the location of the hydrogen bond on lattice dynamics and hence on lowtemperature properties of glasses.
\end{abstract}

Keywords: Glass transition; Monohydroxy alcohols; Specific heat; Polymorphism; Molecular glasses; Crystallization kinetics

\title{
1 Introduction
}

The very nature of glasses (and disordered condensed matter, in general) and of glasstransition related phenomena still represents an open challenge in condensed-matter physics [1] and chemical physics sciences, after more than one hundred years of research on these subjects [2].

In particular, much interest has been recently paid to molecular glass-forming liquids. One practical reason for such an interest is that these liquids usually solidify either into crystal or glassy states at temperatures between liquid-nitrogen and room temperatures, therefore providing easy access to the different states of the substance for many experimental techniques. They often also include manifestations of polymorphism and even polyamorphism [3] (i.e. the apparent existence of first-order transitions between two liquid states of a singlecomponent substance or, more generally, between two distinct amorphous states of that substance), that makes them even more interesting to be studied.

In this paper, we briefly review the main calorimetric and thermodynamic results found in the family of monohydroxy alcohols - or monoalcohols- $\mathrm{H}\left(\mathrm{CH}_{2}\right)_{n} \mathrm{OH}$ for $n=1,2,3$, 4 , both from our own experiments during the last decade and from the literature. Firstly, we will discuss how the gradual elongation of the molecule with increasing number of ethyl groups affects the vitrification vs crystallization kinetics for primary monoalcohols (that is, when the hydrogen bond is fixed at the end of the molecule). We will also address briefly the 
reported presence of crystalline polymorphism in ethanol and polyamorphism in butanol. Then, we will show the noticeable changes that occur in the lattice dynamics and hence in the low-temperature properties of both glass and crystal states when chemical isomers are introduced, namely by comparing 1-propanol vs 2-propanol and 1-butanol vs 2-butanol. Finally, available thermodynamic data of all these monoalcohols, at the glass and crystal melting transitions, will be compiled and globally discussed, checking whether proposed correlations in the literature with liquid fragility could shed light on the observed behaviour.

\section{Primary monohydroxy alcohols: elongating the molecule}

\subsection{Methanol}

Pure methanol, $\mathrm{CH}_{3} \mathrm{OH}$, has a small, 'quasi-spherical' molecule and readily crystallizes, being very difficult to obtain methanol as bulk glass for standard conditions and cooling rates in typical calorimetric experiments [4]. Some researchers have studied methanol with a few molar percent of water as to avoid crystallization, but that is certainly another substance. The structure of both the liquid and the two low-temperature crystal phases found at ambient pressure consists of linear chains of alternating hydrogen bonded molecules [5].

\subsection{Ethanol}

For $n=2$, we have ethanol, $\mathrm{CH}_{3} \mathrm{CH}_{2} \mathrm{OH}$, which is not an excellent glass-former but at least the glass state can be readily obtained by supercooling the liquid at $30 \mathrm{~K} / \mathrm{min}$ [6]. However, somewhat slower cooling rates above $6 \mathrm{~K} / \mathrm{min}$ could suffice [7-11], since the critical cooling rate for ethanol seems to depend on several factors such as minor water impurity, mechanical or geometrical details of the experimental cell driving heterogeneous crystallization and even on the previous thermal history of the liquid [12].

Pure ethanol (see figure 1) exhibits a very rich polymorphism presenting four different solid states [6-8]: the ordinary amorphous solid or glass state when the liquid is cooled sufficiently fast, a body-centred cubic plastic crystal (PC) with rotational disorder which by quenching becomes an orientationally-disordered crystal (ODC), and a fully-ordered (monoclinic) crystal state with 4 atoms per unit cell [13], which in turn exists in four distinct phases [9], depending on temperature and thermal history. Therefore, a relatively simple and easily available chemical substance as ethanol offers an extremely useful benchmark to study the glass transition phenomenon and the very nature of glasses and amorphous solids, since one can choose and discriminate the roles played by translational, rotational and orientational disorder.

\section{[Insert figure 1 about here]}

Very interestingly, both the standard glass transition (amorphous solid $\leftrightarrow$ supercooled liquid) and that dynamical freezing of the PC into the ODC were found to occur at the same temperature $\left(T_{\mathrm{g}} \approx T_{\mathrm{g}}{ }^{\prime} \approx 97 \mathrm{~K}\right)$ and with comparable discontinuities in the specific heat $[6,8]$. Furthermore, the ODC state exhibited, qualitatively and almost quantitatively, the same universal glassy properties at low temperatures such as tunnelling states, the plateau in the thermal conductivity or the boson peak as the truly amorphous, structural glass state $[8,10$, $11,14]$. For those reasons, such ODCs are sometimes also named 'glassy crystals' or 'orientational glasses'. 


\subsection{Propanol}

By putting a third carbon atom (i.e. adding another ethyl group) to the monoalcohol chain, one gets for $n=3$ the best glass-forming substance of the series: 1-propanol or n-propanol. This fact is evidenced by a narrower temperature range for the supercooled liquid than in ethanol. The glass transition temperatures are essentially the same, $T_{\mathrm{g}} \approx 98 \mathrm{~K}$ for 1 -propanol, but the melting point is now $11 \mathrm{~K}$ lower: $T_{\mathrm{m}}=148 \mathrm{~K}$. Indeed complete crystallization of 1 propanol requires annealing over several hours at temperatures within a narrow range about $135 \mathrm{~K}[15,16]$. The crystalline state of 1-propanol was also found to present a monoclinic structure but with 6 atoms per unit cell [16].

\subsection{Butanol}

By further elongating the molecule $(n=4)$, the glass-forming tendency begins to reverse: 1-butanol glass can be easily obtained when cooling the liquid at a moderately slow rate, but it crystallizes when heating the glass or if the supercooled liquid is kept at certain temperatures long enough. Interestingly, a metastable phase (between the glass and crystal ones) was found in 1-butanol [17], very similar to that previously found in triphenyl phosphite (TPP) $[18,19]$. In both cases, a new solid phase denoted as glacial phase or glacial state [18], obtained by a first-order, exothermic transformation from the supercooled liquid state was observed. Among other interpretations, this controversial 'glacial phase' was firstly attributed in TPP to a defect-ordered phase within the theory of frustration-limited domains [18], and later was considered (both for TPP and 1-butanol) as a manifestation of liquid-liquid transitions between two distinct liquid (with their corresponding amorphous solid) states [19]. A brief review about polyamorphism with special emphasis on the case of TPP can be found in Ref. [3].

However, Hédoux et al. [20] have conducted several experiments on TPP, and have shown that the atypical transformation kinetics found is a consequence of a time lag of nucleation, inducing a high nucleation rate which frustrates the crystallization and leads to the so-called "glacial state". By using Raman-scattering, the origin of the frustration of the crystallization in 1-butanol has also been explained [21] from the consideration that the rearrangement of the hydrogen-bonded molecules is unavoidably prevented by the very low molecular mobility of the system only a few degrees above $T_{\mathrm{g}}$. The "glaciation" process would be an abortive crystallization driven by the formation of a layered, two-dimensional hydrogen-bond network. This particular transformation kinetics can nonetheless be described $[20,21]$ by a classical nucleation-growth law. In fact, the temporal evolution of the transformation degree in 1-butanol at two different temperatures above $T_{\mathrm{g}}$ were found [21] to follow a clear Avrami sigmoidal shape, indicative of a first-order character for the isothermal transformation. The authors estimated degrees of crystallinity at the end of the transformation processes typically around 70-80\% [21]. Our recent experiments [22, 23] support this view that the "glacial phase" of 1-butanol or n-butanol is just a mixture of many nanocrystallites embedded in a disordered matrix, essentially due to an aborted crystallization originated by a high nucleation rate in a temperature range where the crystal growth is low. The stable crystalline state was found to present triclinic symmetry with 6 atoms per unit cell [23]. 


\section{Chemical isomerism in monohydroxy alcohols: the role of hydrogen-bond location}

From the case of propanol on, i.e. for $n \geq 3$, a new possibility arises to play with a different variable to study its influence on the physical and chemical behaviour of their corresponding liquid and solid phases, namely the existence of some chemical isomers. The chemical isomer of 1-propanol is 2-propanol or iso-propanol, that is also a good glass-former, with glass transition and melting points temperatures, $T_{\mathrm{g}} \approx 115 \mathrm{~K}$ and $T_{\mathrm{m}}=185 \mathrm{~K}$, surprisingly very much higher than those of 1-propanol. Moreover, 2-propanol remarkably exhibits a much higher boson peak (seen in figure 2 as a broad maximum in the Debye-reduced specific heat $C_{\mathrm{p}} / T^{3}$ ) than in 1-propanol. Interestingly, the same softening of the vibrational lattice was observed in the corresponding crystal states $[11,15]$ with obtained Debye coefficients $C_{\text {Deb }}=$ $1.10 \mathrm{~mJ} \cdot \mathrm{mol}^{-1} \mathrm{~K}^{-4}$ for 1-propanol and $C_{\mathrm{Deb}}=1.55 \mathrm{~mJ} \cdot \mathrm{mol}^{-1} \mathrm{~K}^{-4}$ for 2-propanol. Therefore, both crystalline and glassy states of 2-propanol exhibit a significantly higher density of Debye-like acoustic excitations than those of 1-propanol.

\section{[Insert figure 2 about here]}

On the other hand, 2-butanol is a better glass-former than 1-butanol and we have not been able to obtain its crystalline state. Nevertheless, the specific heat of the glass states of both isomers has been measured [24,25] at low temperatures, and we have found again that the secondary monoalcohol presents a much higher boson peak than the corresponding primary one, as shown in figure 2 (right panel). To change the hydrogen-bonding location from the end to the centre of the molecule clearly soften the low-frequency vibrational spectrum and enhance its density of states, for both Debye-like acoustic phonons and glassy 'soft modes'.

Finally, we want to mention another possible strategy to investigate the microscopic nature of low-frequency excitations in hydrogen-bonded glasses. It is to assess isotopic effects on their physical properties by employing fully- or partially-deuterated samples. Indeed fullydeuterated ethanol has been measured and compared to normal hydrogenated ethanol $[8,10$, 11], but this method has not been fully exploited yet. In those experiments with fullydeuterated ethanol we showed that the lattice-vibrational contributions for all solid states (crystal, glass and ODC) are well accounted for by Debye's theory, since the cubic coefficients of the low-temperature specific-heat $C_{\mathrm{p}}$ data nicely scaled with $M^{3 / 2}$ ( $M$ being the molecular mass), as expected [10]. On the other hand, the height of the broad maximum in $C_{\mathrm{p}} / T^{3}$ (i.e. the boson peak) and the linear term of $C_{\mathrm{p}}$ (i.e. the density of tunneling states) for both the glass and the ODC were found to roughly scale with the inverse square root of the moment of inertia of the molecule [11], as previously suggested by some models. Furthermore, these similar correlations in ethanol for glass and ODC phases suggested a common "orientational" origin for the low-temperature excitations, at least in ethanol. Therefore, it would be especially interesting to compare the dynamical and thermal behaviour of monoalcohol glasses with partial deuteration, by selectively replacing hydrogen atoms by deuterium for bonding $(\mathrm{OH})$ groups and non-bonding (methyl or ethyl) groups. By observing different isotopic effects, depending on the location of the deuterated atoms, on relevant experimental data, one could shed light on the unknown microscopic nature of those anomalous low-energy excitations, responsible for the universal low-temperature properties of glasses. 


\section{Discussion}

As we have shown above, by gradually increasing the 'length' of the molecule for primary monoalcohols, one modifies the balance between crystallization kinetics and glass-forming ability. Whereas pure methanol (a small, 'quasi-spherical' molecule) readily crystallizes and we are not able to obtain it in the glass state for standard conditions and cooling rates, ethanol seems to possess a 'critical' aspect ratio for its molecule, with competing interactions and driving forces which are likely at the origin of its interesting and rich polymorphism, depicted in figure 1. By adding another ethyl group to the hydrocarbon backbone and hence further elongating the 'ellipsoidal' molecule, the best glass-forming primary monoalcohol is observed: 1-propanol. Propanol molecules seem to be long enough as to seriously hinder the crystallization arrangement. With the next in the series, the glass-forming tendency begins to reverse: 1-butanol glass can be easily obtained when cooling the liquid at a moderately slow rate, but it readily crystallizes when heating the glass, though often in a two-step process that could be wrongly taken as a polyamorphic transition, as discussed above in section 2.4 . We have recently performed calorimetric, thermal conductivity, Brillouin-scattering and x-ray diffraction experiments $[22,23]$ which strongly support the alternative interpretation that the "glacial phase" of 1-butanol is just a mixture of many nanocrystallites embedded in a disordered matrix. Specifically, Brillouin spectra [22] of the "glacial phase" were composed of two different phonon-like peaks, one of them clearly being the same acoustic peak of the stable crystal. Furthermore, x-ray diffraction experiments [23] showed that the "glaciation" transformation process corresponded to an emergence of the crystalline Bragg peaks onto the amorphous diffraction pattern. When the "glacial phase" was heated to obtain the stable triclinic crystal state, the main Bragg peaks were observed to lie in exactly the same positions, only that much sharper and better defined than in the previous metastable phase, whereas the amorphous background disappeared and allowed the observation of the secondary peaks of the crystalline diffraction pattern. The size of these nanocrystallites was estimated to be of several tens of nanometre. To which extent our finding about the nanocrystalline nature of the so-called glacial state of 1-butanol can be applied to other cases such as TPP remains to be investigated, though earlier works [20] gave evidence in the affirmative.

Interestingly, this glass-forming tendency found in primary monohydroxy alcohols is exactly the same previously observed for the first four members of the alkyl-cyclohexanes family [26]. It suggests therefore that when the hydrocarbon molecules are long enough, they begin to find easier to align themselves and crystallize, and this crossover typically occurs when passing from 3 to 4 carbon atoms in the linear chain.

On the other hand, we have compiled in table 1 the most relevant calorimetric and thermodynamic data concerning the glass transition and the crystal melting for all the considered primary and secondary monoalcohols up to $n=4$, as well as reported values for the so-called fragility index [27] ascribed to average relaxation times $\tau$ obtained from either viscosity or dielectric measurements. The fragility or steepness index $m$ is defined as the slope of a fragility plot $\log _{10}<\tau>$ vs $T_{\mathrm{g}} / T$ evaluated at $T_{\mathrm{g}}$ and is usually considered as an essential characteristic of the kinetic and relaxational behaviour of undercooled liquids. Many attempts have been made to correlate these kinetic properties of undercooled liquids with their thermodynamic behavior around the glass transition. Among others, an empirical relation recently suggested $[27,28]$ tried to correlate the fragility $m$ with a dimensionless combination of the specific-heat discontinuity measured at the glass transition temperature $\Delta C_{\mathrm{p}}\left(T_{\mathrm{g}}\right)$ and the melting enthalpy $\Delta H_{\mathrm{m}}$, through the equation: 
Alternatively, by making use of the typical rule $T_{\mathrm{g}} / T_{\mathrm{m}} \approx 2 / 3$, one could approximately write [27]

$$
m=40 \frac{\Delta C_{p}\left(T_{g}\right)}{\Delta S_{m}}
$$

where $\Delta S_{\mathrm{m}}$ is the entropy of melting, thereby establishing a direct correlation between a kinetic fragility and a thermodynamic one. Based upon the random first-order transition (RFOT) theory, Lubchenko and Wolynes [34] found a relation very similar to equations (1) and (2), with only slightly smaller numerical prefactors, hence giving theoretical support to that empirical finding. In Ref. [27], Wang et al. found a good correlation between kinetic and thermodynamic fragilities for more than 50 nonpolymeric glass-forming materials, with a very few exceptions. As can be seen in table 1, primary and secondary monohydroxy alcohols exhibit intermediate values of fragility and, more importantly, such a correlation is not observed at all. Furthermore, one would expect a lower fragility index (i.e. a stronger glassforming liquid) for the case of propanol, the best glass former, and the opposite trend for methanol. This is clearly not the case, neither for the (kinetic) measured fragility nor for the (thermodynamic) calculated one. This failure of the general correlation found in other glassforming systems may be traced back to the earlier idea by Angell [35], that the intermediate fragility for the viscosity found in hydrogen-bonded liquids could be the compensating result of a very strong kinetic behaviour (with high energy barriers between adjacent minima in the energy landscape) and a very fragile thermodynamic behaviour (with a high density of configurational states accounting for the large values of $\Delta C_{\mathrm{p}}\left(T_{\mathrm{g}}\right)$ typically found in alcohols).

The kinetic-thermodynamic correlation fails even more dramatically for the glass-like transition ODC-PC in ethanol, which in other respects is very similar to the canonical glass transition. The enthalpy of melting for plastic crystals is always very low and consequently they are predicted by eq. (1) to be very fragile, what is in clear disagreement with the results found from dielectric measurements in ethanol of the relaxation time, giving $m=60$ for the structural glass and only $m=40$ for the plastic crystal [29]. In those cases, as well as for the fragility indices $m_{\text {meas }}$ of 1-butanol [30] and 2-butanol [33] in table 1, the relaxation-time curves have been determined from the dielectric-loss peak positions of the non-Debye, primary $(\alpha)$ relaxation process, that is the one normally attributed to the glass transition and hence equivalent to the relaxation times obtained from viscosity measurements.

Another interesting concept traditionally used to rationalize the thermodynamic magnitudes for the glass transition and crystal melting processes is that of the number of "beads", i.e. more or less spherical and compact units that constitute the molecule and account for their configurational degrees of freedom. Wolynes and co-workers [34, 36] have proposed to count beads from the entropy of melting. In particular, they found an entropy of fusion per particle for Lennard-Jones spheres, $s_{\mathrm{LJ}}=1.68 k_{\mathrm{B}}$, and therefore they proposed a general relation 


$$
N_{\text {beads }}=\Delta S_{\mathrm{m}} / 1.68 R
$$

The corresponding estimations of the number of configurational beads per molecule for the monohydroxy alcohols are also shown in table 1. Using eq. (3), Stevenson and Wolynes [36] reported calculated values of 1.3 for methanol, 2.3 for ethanol and 2.6 for 1propanol, whereas we have determined values of 1.6, 2.2 and 2.6, respectively, the small differences arising from the different sources of experimental data employed for the entropy of melting. In addition, we obtain 3.6 beads per molecule of 1-butanol. There is therefore a reasonable increase of $N_{\text {beads }}$ with increasing chain length (basically, the number of carbon atoms) for primary alcohols, but this trend is broken for secondary alcohols, where the intermolecular connections change significantly. Eq. (3) obviously includes several approximations and oversimplifications, and one should not expect to obtain exact integer numbers. In the RFOT theory, the entropy of fusion comes in only as a way of relating the entropy costs of localizing real molecules that are not spherical to their locations in the crystal and the entropy loss for localizing "beads" which are assumed to be spheres.

In contrast, the effective number of beads for the particular case of the plastic crystal (usually ascribed to the rotation of the methyl group) would be only of around 1 bead per 3 molecules of ethanol. This small number is due to the very small enthalpy of fusion typical of plastic crystals.

\section{Conclusions}

Putting together our more recent specific-heat measurements and calorimetric studies of butanol isomers with our earlier experiments on ethanol and propanol, as well as with other data from the literature, we have been able to discuss how either the gradual elongation of the monoalcohol molecule with increasing number of ethyl groups or the hydrogen-bonding location have a strong influence on the glass-forming ability, as well as on low-frequency dynamics and low-temperature properties of molecular solids.

The competition between crystallization rate and glass-forming ability seems to depend mainly on the aspect ratio of the molecules, ranging from the easiest crystal former methanol to the best glass former propanol. Intermediate glass formers as ethanol or butanol has been argued to present the added value of a rich polymorphism -the former- and the possibility of comparing different chemical isomers - the latter-. In particular, it was found that the boson peaks of the structural (amorphous) and orientational (ODC) glasses of ethanol were very similar, whereas those of secondary alcohols were unexpectedly larger than those of their corresponding primary alcohols.

By comparing kinetic and thermodynamic data for this model system of monohydroxy alcohols, we have found that proposed phenomenological correlations for the fragility of undercooled liquids is not fulfilled at all for these -and probably others- hydrogen-bonded liquids, which seem to behave kinetically as strong liquids but thermodynamically as fragile ones. For them, the entropy of crystal melting and the specific-heat jump at the glass transition do not directly correlate with the kinetic fragility index of the supercooled liquid. Furthermore, the glassy transition for the plastic crystal (i.e. the dynamical freezing of rotational disorder into static orientational disorder), that behaves so similarly to the canonical glass transition in many other respects, exhibits a completely disparate behaviour. 


\section{Acknowledgments}

We acknowledge financial support by the Spanish Ministry of Science within both project FIS2009-09811 and program CONSOLIDER Nanociencia Molecular CSD200700010 .

\section{References}

[1] P. W. Anderson, Science 267, 1615 (1995).

[2] A non-exhaustive list of good review textbooks is: (a) R. Zallen, The Physics of Amorphous Solids, (Wiley, 1983); (b) S. A. Brawer, Relaxation in viscous liquids and glasses (American Ceramics Society, 1983); (c) S. R. Elliott, Physics of Amorphous Materials, 2nd ed. (Longman, 1990); (d) I. Gutzow and J. Schmelzer, The Vitreous State: Thermodynamics, Structure, Rheology, and Crystallization (Springer, Berlin-Heidelberg, 1995); (e) P. G. Debenedetti, Metastable Liquids: Concepts and Principles (Princeton, 1996); (f) E.-J. Donth, The Glass Transition: Relaxation Dynamics in Liquids and Disordered Materials (Springer, 2001); (g) K. Binder and W. Kob, Glassy materials and disordered solids (World Scientific, Singapore, 2005); (h) L. Leuzzi and Th. M. Nieuwenhuizen, Thermodynamics of the Glassy State (Taylor and Francis, 2007).

[3] J. Senker and E. Rössler, Chem. Geol. 174, 143 (2001).

[4] M. Sugisaki, H. Suga and S. Seki, Bull. Chem. Soc. Jpn. 41, 2586 (1968).

[5] K. J. Tauer and W. N. Lipscomb, Acta Crystallogr. 5, 606 (1952); B. H. Torrie, S. X. Weng and B. M. Powell, Mol. Phys. 67, 575 (1989).

[6] O. Haida, H. Suga and S. Seki, J. Chem. Thermodyn. 9, 1133 (1977).

[7] A. Srinivasan, F. J. Bermejo, A. de Andrés, J. Dawidowski, J. Zúñiga and A. Criado, Phys. Rev. B 53, 8172 (1996).

[8] M. A. Ramos, S. Vieira, F. J. Bermejo, J. Dawidowski, H. E. Fisher, H. Schober, M. A. González, C. K. Loong and D. L. Price, Phys. Rev. Lett. 78, 82 (1997).

[9] M. A. Ramos, I. M. Shmyt'ko, E. A. Arnautova, R. J. Jiménez-Riobóo, V. RodríguezMora, S. Vieira and M. J. Capitán, J. Non-Cryst. Solids 352, 4769 (2006).

[10] C. Talón, M. A. Ramos and S. Vieira, Phys. Rev. B 66, 012201 (2002).

[11] M. A. Ramos, C. Talón, R. J. Jiménez-Riobóo and S. Vieira, J. Phys.: Condens. Matter 15, S1007 (2003).

[12] B. Kabtoul, R. J. Jiménez-Riobóo and M. A. Ramos, Phil. Mag. 88, 4197 (2008).

[13] P.G. Jönsson, Acta Crystallogr. Sect. B 32, 232 (1976). 
[14] A. I. Krivchikov, A. N. Yushchenko, V. G. Manzhelii, O. A. Korolyuk, F. J. Bermejo, R. Fernández-Perea, C. Cabrillo, and M. A. Gonzalez, Phys. Rev. B 74 (2006) 060201.

[15] C. Talón, M. A. Ramos, S. Vieira, I. Shmyt'ko, N. Afonikova, A. Criado, G. Madariaga and F. J. Bermejo, J. Non-Cryst. Solids 287, 226 (2001).

[16] C. Talón, F. J. Bermejo, C. Cabrillo, G. J. Cuello, M. A. González, J. W. Richardson, Jr., A. Criado, M. A. Ramos, S. Vieira, F. L. Cumbrera and L. M. González, Phys. Rev. Lett. 88, 115506 (2002).

[17] B. V. Bol'shakov and A. G. Dzhonson, Dokl. Phys. Chem. 393, 318 (2003); B. V. Bol'shakov and A. G. Dzhonson, J. Non-Cryst. Solids 351, 444 (2005).

[18] A. Ha, I. Cohen, X. Zhao, M. Lee and D. Kivelson, J. Phys. Chem. 100, 1 (1996).

[19] H. Tanaka, R. Kurita and H. Mataki, Phys. Rev. Lett. 92, 025701 (2004); R. Kurita and H. Tanaka, Science 306, 845 (2004); R. Kurita and H. Tanaka, J. Phys.: Condens. Matter 17, L293 (2005).

[20] A. Hédoux, Y. Guinet, M. Foulon, and M. Descamps, J. Chem. Phys. 116, 9374 (2002).

[21] A. Wypych, Y. Guinet and A. Hédoux, Phys. Rev. B 76, 144202 (2007).

[22] M. Hassaine, R. J. Jiménez-Riobóo, I. V. Sharapova, O. A. Korolyuk, A. I. Krivchikov and M. A. Ramos, J. Chem. Phys. 131, 174508 (2009).

[23] I. M. Shmyt'ko, R. J. Jiménez-Riobóo, M. Hassaine and M. A. Ramos, J. Phys.: Condens. Matter 22, 195102 (2010).

[24] A. I. Krivchikov, M. Hassaine, I. V. Sharapova, O. A. Korolyuk, R. J. Jiménez-Riobóo and M. A. Ramos, J. Non-Cryst. Solids (accepted for publication).

[25] M. Hassaine and M. A. Ramos (unpublished).

[26] A. Mandanici, M. Cutroni, A. Triolo, V. Rodriguez-Mora and M. A. Ramos, J. Chem. Phys. 125, 054514 (2006).

[27] L.-M. Wang, C. A. Angell and R. Richert, J. Chem. Phys. 125, 074505 (2006) and references therein.

[28] L.-M. Wang and C. A. Angell, J. Chem. Phys. 118, 10353 (2003).

[29] S. Benkhof, A. Kudlik, T. Blochowicz and E. Rössler, J. Phys.: Condens. Matter 10, 8155 (1998).

[30] T. El Goresy and R. Böhmer, J. Chem. Phys. 128, 154520 (2008).

[31] D.R. Lide (Editor), Handbook of Chemistry and Physics (CRC Press, Boca Raton, FL, $1992,73^{\text {rd }}$ edition). 
[32] R. J. L. Andon, J. E. Connett, J. F. Counsell, E. B. Lees and J. F. Martin, J. Chem. Soc. (A), 661 (1971).

[33] B. Jakobsen, C. Maggi, T. Christensen and J. Dyre, J. Chem. Phys. 129, 184502 (2008).

[34] V. Lubchenko and P. G. Wolynes, J. Chem. Phys. 119, 9088 (2003).

[35] C. A. Angell, in Hydrogen-Bonded Liquids, J. C. Dore and J. Teixeira (eds.), 59 (1991).

[36] J. D. Stevenson and P. G. Wolynes, J. Phys. Chem. B 109, 15093 (2005). 


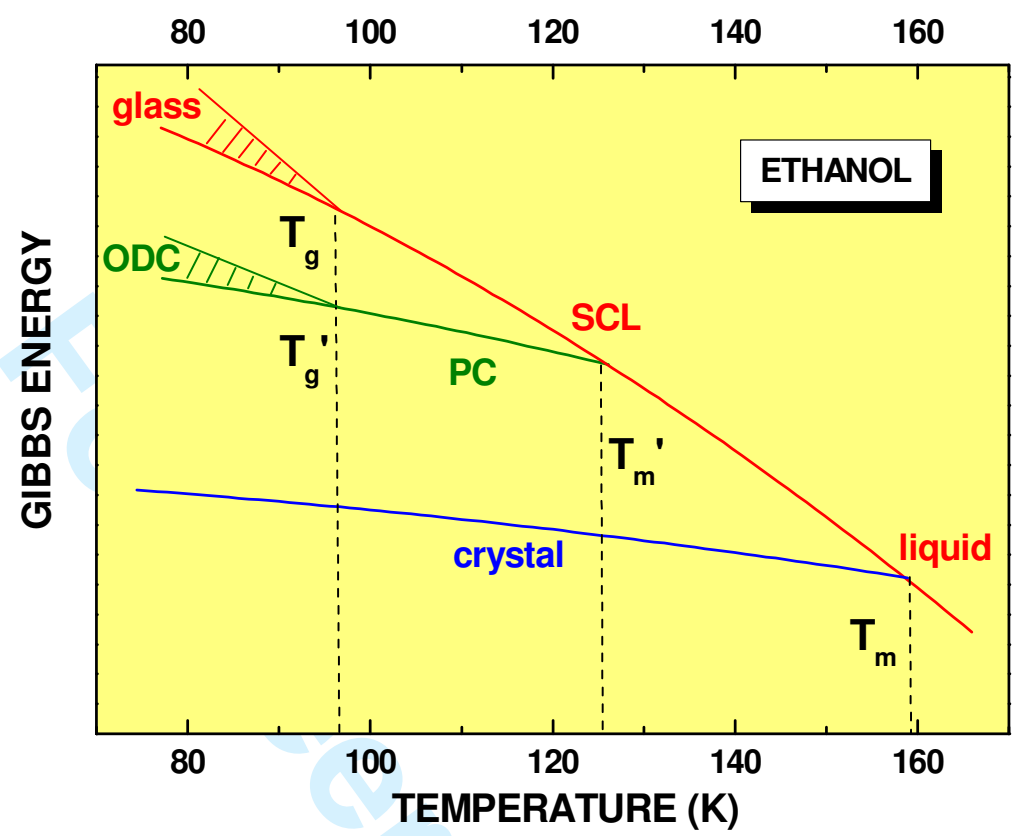

Figure 1. Schematic Gibbs-energy phase diagram for ethanol. When the supercooled liquid (SCL) below the melting point $\left(T_{\mathrm{m}}=159 \mathrm{~K}\right)$ is quenched fast enough, the glass state is obtained at $T_{\mathrm{g}} \approx 97 \mathrm{~K}$. By heating the glass above $T_{\mathrm{g}}$ or by cooling the SCL more slowly, a plastic crystal (PC) is obtained, which becomes an orientationally-disordered crystal (ODC) below a glass-like transition at $T_{\mathrm{g}}{ }^{\prime} \approx 97 \mathrm{~K}$. The $\mathrm{PC}$ melts at around $T_{\mathrm{m}}{ }^{\prime} \approx 125 \mathrm{~K}$ and immediately transforms into a fully-ordered monoclinic crystal. 

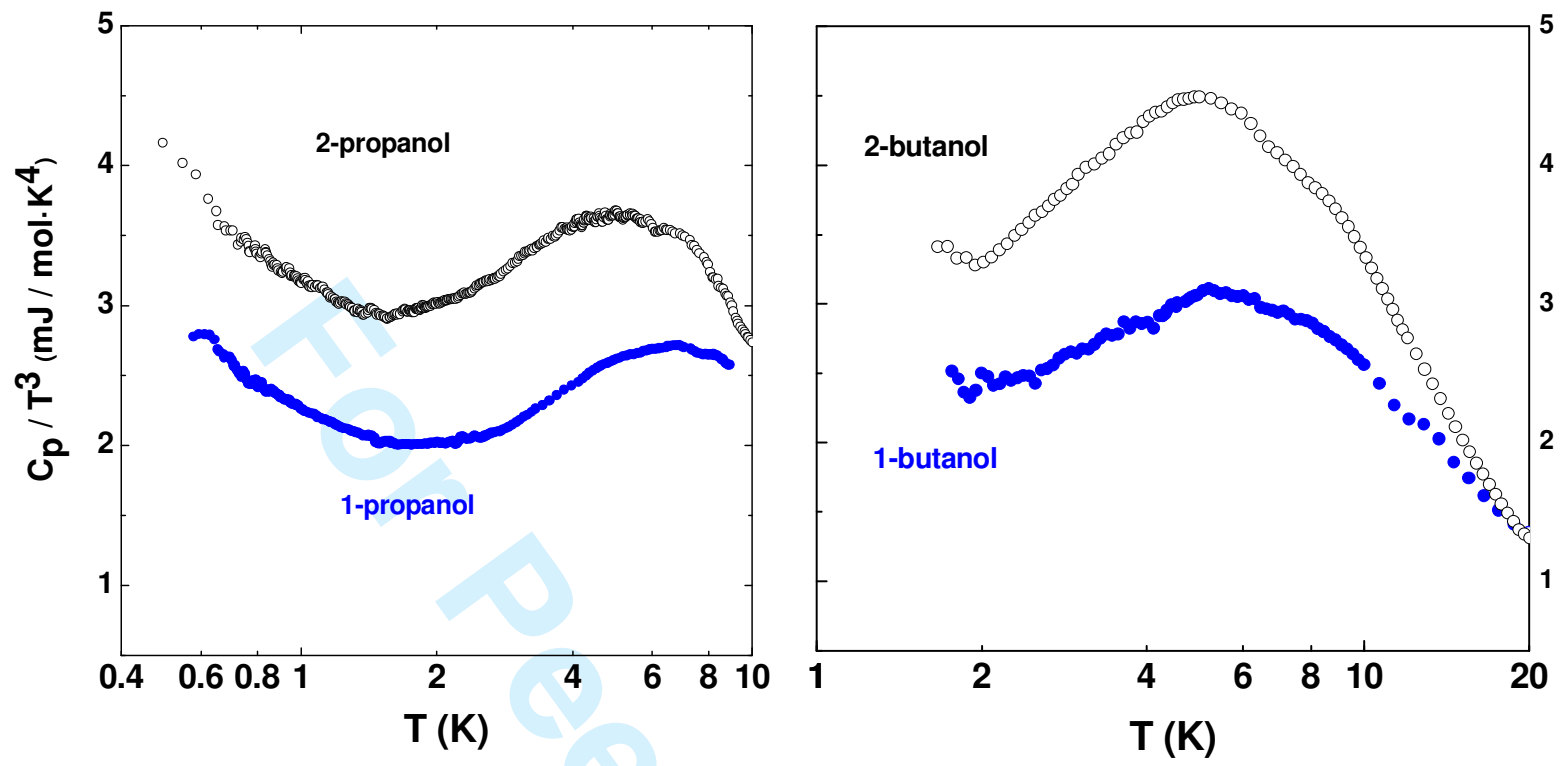

Figure 2. Reduced specific heat $C_{\mathrm{p}} / T^{3}$ for glassy states of 1- and 2-propanol (left panel) and of 1- and 2-butanol (right panel). Note different temperature ranges for the two plots. The boson peak for the secondary alcohol is clearly higher than the corresponding one for the primary alcohol in both cases. 
Table 1. Calorimetric and thermodynamic data of simplest monohydroxy alcohols. $T_{\mathrm{g}}$ is glass-transition temperature and $\Delta C_{\mathrm{p}}\left(T_{\mathrm{g}}\right)$ its specific-heat discontinuity; $T_{\mathrm{m}}$ and $\Delta H_{\mathrm{m}}$ are temperature and enthalpy of melting, respectively; $m_{\text {meas }}$ and $m_{\text {calc }}$ are the measured and calculated (using eq. (1)) values for the fragility index; $N_{\text {beads }}$ is the estimated number of configurational units or "beads" (see text) as defined by eq. (3).

\begin{tabular}{lcccccccc}
\hline substance & $\boldsymbol{T}_{\mathbf{g}}(\mathbf{K})$ & $\begin{array}{c}\Delta \boldsymbol{C}_{\mathbf{p}}\left(\boldsymbol{T}_{\mathbf{g}}\right) \\
\left(\mathbf{J ~ m o l}^{-1} \mathbf{K}^{-\mathbf{1}}\right)\end{array}$ & $\boldsymbol{T}_{\mathbf{m}}(\mathbf{K})$ & $\begin{array}{c}\Delta \boldsymbol{H}_{\mathbf{m}} \\
\left(\mathbf{k J ~ m o l}^{\mathbf{1}}\right)\end{array}$ & $\boldsymbol{m}_{\text {meas }}$ & $\boldsymbol{m}_{\text {calc }}$ & $\boldsymbol{N}_{\text {beads }}$ & Refs. \\
\hline methanol & 103 & 30 & 176 & 3.85 & 39 & 45 & 1.6 & 4,27 \\
ethanol (glass) & 97 & 35.3 & 159 & 4.93 & $55-60$ & 39 & 2.2 & $6,12,27,29$ \\
1-propanol & 98 & 45 & 148 & 5.40 & 40 & 46 & 2.6 & 15,27 \\
1-butanol & 111 & 48 & 184 & 9.28 & 59 & 32 & 3.6 & 22,30 \\
\hline ethanol (ODC) & 97 & 22.8 & 127.5 & 0.66 & 40 & 188 & 0.37 & $6,12,29$ \\
\hline 2-propanol & 115 & 46 & 185 & 5.37 & $?$ & 55 & 2.1 & 15,31 \\
2-butanol & 118 & 39 & 185 & 5.97 & 63 & 43 & 2.3 & $25,32,33$ \\
\hline
\end{tabular}

